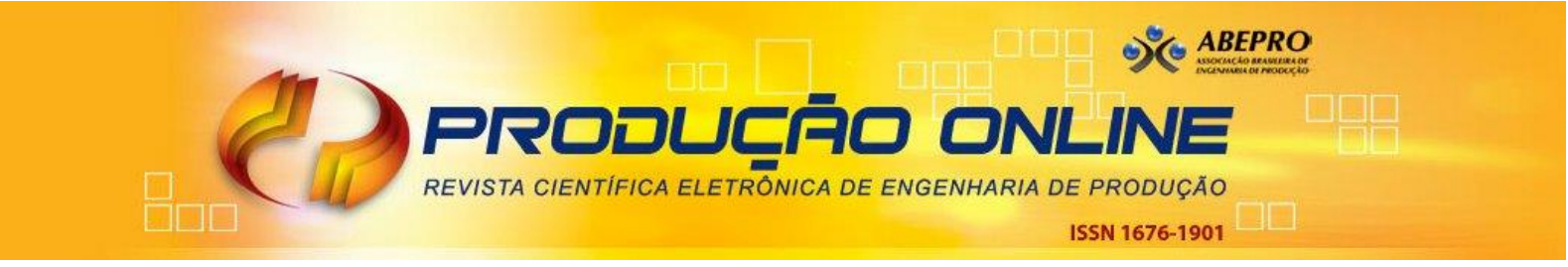

\title{
APOIO À DECISÃO MULTICRITÉRIO NA PRIORIZAÇÃO DE ROTAS PARA O TRANSPORTE URBANO
}

\section{MULTICRITERIA DECISION SUPPORT ON PRIORITIZING ROUTES FOR URBAN TRANSPORT}

\author{
Lorena Hernández*E-mail: lorenahmastrapa@gmail.com \\ Maria Rita Pontes* E-mail: maria.assumpcao@unimep.br \\ Maria Célia de Oliveira* E-mail: maria.oliveira32@unimep.br \\ Daniel René Tasé Velázquez* E-mail: daniel.velasquez@a.unimep.br \\ *Universidade Metodista de Piracicaba (Unimep), Piracicaba, SP
}

Resumo: Dentre os grandes desafios que o fenômeno da rápida urbanização impõe encontra-se o do planejamento de transporte urbano. Com isto, é preciso criar estratégias que possibilitem boas escolhas a fim de dar um melhor conforto ao usuário e funcionários deste serviço. Um dos problemas mais recorrentes é a definição de rotas, considerando a capacidade de atendimento com maior quantidade de pontos de demandas e minimizando o tempo e comprimento do percurso. É muito relevante para o planejamento do transporte urbano, a decisão de quais rotas são as melhores, de acordo com todos esses critérios. Como auxílio à seleção e priorização de rotas de ônibus urbanos, este trabalho propõe um modelo baseado no método multicritério de apoio a decisão, ELECTRE I. Para esta análise, 4 alternativas de rotas foram estudadas considerando 5 critérios de desempenho, a saber: Comprimento do conjunto de rotas; Tempo de viagem (ida e volta) no conjunto de rotas; Demanda potencial do conjunto de rotas; Capacidade do trecho mais carregado do conjunto de rotas e Capacidade do trecho mais carregado do conjunto de rotas. Este modelo é aplicado em uma rede de transporte urbano, já estudado em pesquisas anteriores. Como resultado, a alternativa 1 se destaca como a mais relevante, apresentando o menor tempo de comprimento e tempo de viagem, atendendo à maior demanda potencial dentre todas as rotas.

Palavras-chave: Decisão multicritério. Problema de Transporte Público Urbano. ELECTRE I.

Abstract: Among the major challenges that rapid urbanization phenomenon imposes we found the urban transport planning. In this regard it important to create strategies that allow good choices in to give a better comfort to the users and employees of this service. One of the most recurrent problems is the routes definition, considering the attendance capability with greater quantity of demand points and minimizing the route time and length. It is relevant for the urban transport planning process, to make the decision of which routes are the best, according to all these criteria. Intending to contribute to the selection and prioritization of urban bus routes, this paper proposes a model based on the multicriteria decision support method, ELECTRE I. To carry out this analysis, four alternative routes were studied considering 5 performance criteria, namely: Routes set length; Travel time (round trip) in the route set; Potential demand of the routes set; Capacity of the most loaded segment of the route set and Capacity of the most loaded portion of the route set. This model is applied in an urban transport network, already studied in previous researches. As a result, alternative 1 stands out as the most relevant, presenting the shortest length and travel time, considering the greater potential demand among all routes.

Keywords: Multicriteria Decision. Urban Public Transport Problem. ELECTRE I. 


\section{INTRODUÇÃO}

O crescimento das cidades em termos de superfície e população é uma das tendências globais desde o século passado. $O$ ano 2007 marcou uma etapa histórica no mundo. Neste ano o percentual da população urbana excedeu pela primeira vez a população rural, segundo o relatório das Nações Unidas (2014, p.7).

No contexto do transporte público urbano, o crescimento das cidades no Brasil, nas últimas décadas, trouxe um impacto negativo no nível de serviço à população. Isto é decorrência, na maioria das grandes cidades, pela oferta de linhas de ônibus sem planejamento adequado, gerando maiores custos de operação e desconforto aos usuários.

O crescimento das cidades gera o surgimento de mais polos de negócios e serviços, aumentando a quantidade de destinos das viagens. Passageiros passam a realizar maior número de transbordos na área central. Como consequência, são maiores as penalidades associadas a estas transferências. A solução proposta pela Agência Nacional de Transportes Terrestres - ANTT é a implantação de um sistema integrado com objetivo de otimizar os recursos utilizados no transporte urbano, aumentar a acessibilidade da população, racionalizar o uso do espaço viário e melhorar a qualidade de vida e condições para preservação ambiental (ANTP, 1999, p. 82).

O planejamento do transporte rodoviário baseia-se na construção de grandes vias expressas, priorizando o transporte individual em detrimento do coletivo, caracterizando-se pela desarticulação com o planejamento urbano. Esta falta de sincronismo deu origem a graves problemas de mobilidade nas cidades brasileiras (IPEA, 2016, p.83).

O agravamento dos problemas de mobilidade, resultantes deste modo fragmentado de ver a cidade e seus sistemas de transportes, levou ao desenvolvimento de um novo paradigma para a mobilidade urbana. A busca deste paradigma tem recebido diferentes denominações: transporte sustentável, mobilidade sustentável, transporte humano, mobilidade cidadã, entre outros (Brasil, 2005 , p. 26). Este novo padrão propõe melhoria na qualidade de vida das pessoas, resultado de cidades mais acessíveis, com menor desigualdade social e que 
respeitem o meio ambiente. Isto se dá necessariamente no contexto de um tratamento sistêmico e integrado da mobilidade. No entanto, as cidades são sistemas complexos - evoluindo constantemente e o planejamento para um futuro sustentável não é uma tarefa fácil. O domínio do transporte terá de sofrer grandes mudanças estruturais e comportamentais para se adaptar ao futuro como esperado neste paradigma. A maior deficiência no planejamento de transporte coletivo urbano, do ponto de vista da mobilidade nas cidades do Brasil, é a ausência de uma rede metropolitana integrada de transportes que atenda a demanda da cidade aportando benefícios para toda a população.

Este trabalho enquadra-se no tema do transporte urbano sustentável com a proposição de método que visa tornar o sistema de transporte urbano mais acessível, priorizando o transporte público que permita deslocar as pessoas de forma econômica, rápida, confortável e saudável.

Espera-se com este método, contribuir para a construção de um sistema cientificamente projetado que possa reduzir o total de ônibus requeridos para transportar um determinado volume de passageiros, viabilizando o uso mais eficiente e econômico da frota disponível. Tal sistema gera ainda outros benefícios, como: redução em congestionamentos e redução da sobrecarga do ônibus com resultante aumento da segurança, conforto e velocidade no transporte coletivo urbano. O método baseia-se no conceito de sistema integrado por ônibus. Os sistemas integrados por ônibus, também chamados de sistemas tronco-alimentados de transporte coletivo urbano, contam com terminais de integração e linhas de características alimentadoras. Estes sistemas proporcionam deslocamentos entre os bairros periféricos e o terminal, além de linhas troncais pela integração nos terminais. Isto permite que o usuário se desloque em corredores de tráfego em veículos de maior capacidade até a região central do município (AVELAR, 2011, p.2).

O método apresentado objetiva selecionar a alternativa de rotas que seja mais rentável para a conexão de uma rede de transporte público urbano. Para isso, são analisadas várias rotas alternativas com um modelo multicritério de apoio a decisão, ELECTRE I. O uso da abordagem multicritério justifica-se, pois, a resolução do problema de transporte urbano leva em consideração múltiplos critérios 
conflitantes, não representados por uma mesma unidade de medida. Para o processamento dos dados foi utilizado o software RStudio. Esta escolha foi devido a rapidez de entrada e processamento dos dados.

O artigo está composto por esta introdução, com a apresentação da problemática do estudo e do propósito do trabalho. A segunda seção descreve o problema PPRTPU (Problema de Projeto de Rotas para o Transporte Público Urbano), indicando abordagens para sua solução. $\mathrm{Na}$ terceira, são analisados os modelos de tomada de decisão multicritério, justificando o modelo escolhido para resolver o problema objeto de estudo. A quarta seção apresenta o desenvolvimento do modelo para o caso de estudo, apresentando os resultados obtidos. Finalmente são expostas as conclusões do trabalho.

\section{DESCRIÇÃO DO PROBLEMA PPRTPU}

Segundo Dantzig et al. (1979), o PPRTPU refere-se à construção de novas vias de tráfego ou a expansão da capacidade de vias existentes. Esta definição é bastante comum na literatura para o catálogo de problemas gerais de desenho de rede de transporte. Já Friesz (1985), define que o PPRTPU visa determinar as localizações ótimas de instalações a serem adicionadas a uma rede de transporte, ou indicar melhorias de capacidade de instalações existentes. Nesta definição, as instalações podem ser representadas por nós ou por links.

Magnanti e Wong (1984), corroborando com os autores acima, ampliam a visão deste problema. Eles afirmam que o PPRTPU lida com uma hierarquia completa de processos de tomada de decisão no planejamento de transporte, incluindo decisões estratégicas, táticas e operacionais. As decisões estratégicas são decisões em longo prazo relacionadas com infraestrutura de redes de transporte, incluindo redes de transporte público e de localização de instalações. As decisões táticas, tomadas entre um prazo mediano e curto, são relativas à utilização eficaz da infraestrutura e de recursos das redes de transporte urbano existentes. As decisões operacionais são decisões de curto prazo, principalmente relacionadas ao controle de fluxo de tráfego, gerenciamento de demanda ou problemas de programação das operações do serviço de transporte. A figura 1 resume o processo de solução do 
PPRTPU, mostrando sua complexidade e a interdependência de seus subproblemas. É importante destacar que a resolução de cada subproblema influi sobre os demais subproblemas e no PPRTPU como um todo. Além disso, os pontos de entrada de informações podem ser comuns para subproblemas que compõem 0 PPRTPU.

Figura 1 - Exemplo de um mapa do processo de solução do PPRTPU

Entrada

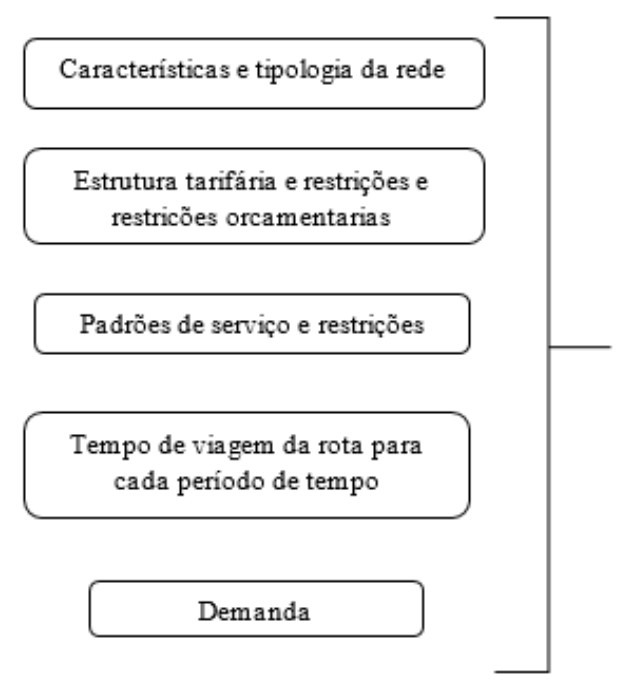

Saida

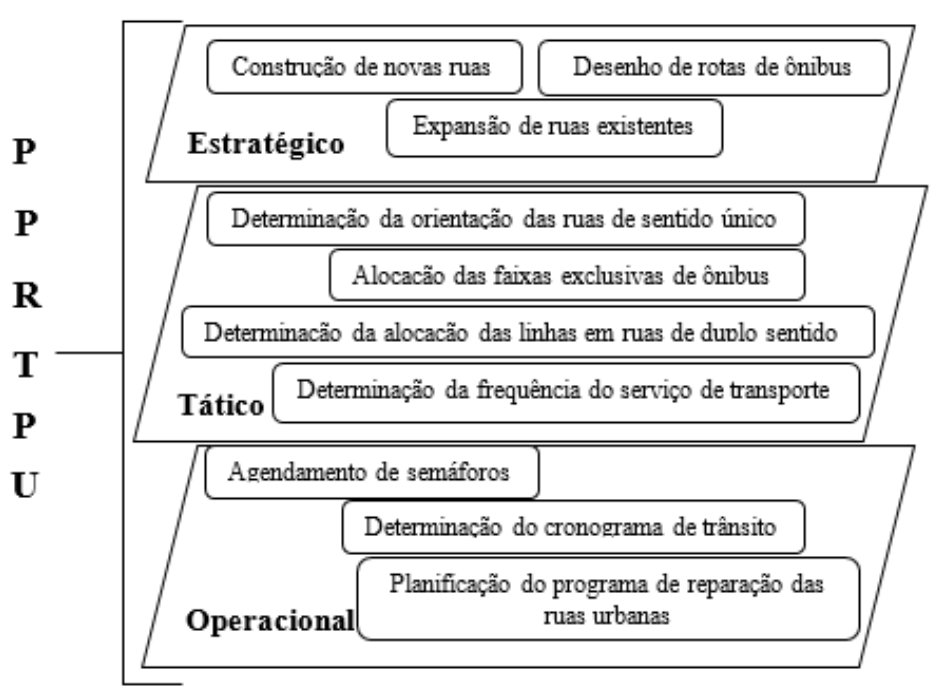

Fonte: Adaptado de Farahani et al. (2013); Ibarra-Rojas, López-Irarragorri e Rios-Solis (2015).

Devido à complexidade e abrangência do problema muitos estudos são dedicados a resolver um subproblema, como é o caso do presente estudo.

\subsection{Modelos e abordagens para solução do PPRTPU}

A modelagem do PPRTPU inclui aspectos relacionados ao problema: (1) objetivos; (2) parâmetros tais como, estrutura da rede rodoviária, padrões e características da demanda de viagens dos passageiros, estratégias de operação que possam melhorar a capacidade e o desempenho do sistema de Transporte Público, bem como as limitações do problema; (3) técnicas de solução que podem ser exatas ou heurísticas (KEPAPTSOGLOU; KARLAFTIS, 2009).

Chakroborty (2003) destacou a dificuldade na formulação matemática de um PPRTPU, uma vez que é inerentemente discreto e conceitos como transferências e continuidade de rota são difíceis de representar. Baaj e Mahmassani (1991) 
discutiram a complexidade do problema como decorrente de sua natureza combinatória, não-linear, não-convexidade e multiobjetivo. Eles também descreveram as dificuldades na formulação como um modelo matemático pela indefinição de layouts de rotas espaciais aceitáveis. Diante disso, foram propostas várias abordagens para solucionar o PPRTPU, tais como: otimização discreta, aproximação contínua, programação não linear (exemplos de abordagens analíticas) e procedimentos heurísticos, como os métodos usados no caso em estudo. Devido a complexidade do problema, a busca de sua solução, normalmente, utiliza uma estratégia de decomposição em subproblemas.

O PPRTPU pode ser modelado como um problema de otimização discreta para determinar o conjunto de linhas para uma dada matriz Origem-Destino, considerando os tempos de viagem previstos por link na rede. Uma alternativa a esta abordagem é representar o PPRTPU por modelos que façam análise utilizando abordagens de aproximação contínua. Neste caso, a demanda de passageiros é representada como uma função contínua sobre um espaço geográfico em vez de uma matriz Origem-Destino (DAGANZO, 2010; OUYANG et al., 2014). A solução de uma aproximação contínua é baseada em uma representação idealizada da cidade que considera estruturas de grades específicas (retangulares, circulares, hub-espokes, por exemplo) (IBARRA-ROJAS, LÓPEZ-IRARRAGORRI; RIOS-SOLIS, 2015). Segundo Kepaptsoglou e Karlaftis (2009), as abordagens analíticas desenvolvem relações entre os componentes da rede de transporte público representada com estruturas idealizadas.

Outra abordagem alternativa do PPRTPU modela as decisões de atribuição de passageiros usando restrições não-lineares ou funções objetivas não-lineares, levando a problemas não convexos (que podem ser resolvidos por algoritmos heurísticos) (IBARRA-ROJAS, LÓPEZ-IRARRAGORRI; RIOS-SOLIS, 2015).

Kepaptsoglou e Karlaftis (2009) resumiram os objetivos práticos da PPRTPU como:

- Maximização do benefício do usuário em Transporte Público, refletindo melhoria na qualidade do serviço, tais como: minimização de custos, de número de viagens e de acessos, de tempo de espera e de transferências; maximização da cobertura de serviços. 
- Já os benefícios do sistema são representados pela máxima utilização da capacidade e minimização de custos de operador de transporte com maximização de lucro: minimização de tamanho de frota, do comprimento da rota, de horas de operação de veículo e de consumo de combustível. Há também objetivos que expressam maximização do uso da capacidade nas rotas da rede de Transporte Público.

- Maximização do bem-estar geral que é representado pela minimização dos custos para os passageiros e para os operadores e conservação de energia - proteção do ambiente contra emissões poluentes e ruído.

- Otimização de parâmetros específicos, como número máximo permitido de rotas na rede de Transporte Público, comprimento de rotas de Transporte Público, bem como o fator de carga, entendido como a taxa de ocupação dos veículos.

Tem-se, então, que as formulações matemáticas para o projeto de rede de Transporte Público são geralmente intratáveis por abordagens exatas / analíticas. No entanto, é possível resolvê-los de forma exata, nos casos, por exemplo, de projeto de linha única (GUAN, YANG, WIRASINGHE, 2003), na presença de simplificações que reduzem o tamanho da rede de Transporte Público (LOWNES; MACHEMEHL, 2010) e para redes de transporte de pequena dimensão (WAN; LO, 2003). As heurísticas e meta-heurísticas avançadas são implementadas, na maioria das vezes, para resolver problemas de PPRTPU. Muitos deles são formulados com modelos de dois níveis para considerar decisões de atribuição de Transporte Público (IBARRA-ROJAS, LÓPEZ-IRARRAGORRI; RIOS-SOLIS, 2015).

Este trabalho utiliza um método de apoio á tomada de decisão multicritério, como complemento ao procedimento de Mastrapa, Leal e Pessoa (2017). Este último foi baseado em procedimento heurístico.

\section{DECISÃO MULTICRITÉRIO}

Decisão multicritério refere-se a tipos de problemas de decisão, considerando questões de natureza econômica, industrial, política e até mesmo social (Cunha, 2003). A decisão pode ser compreendida como um processo de colher informações, 
avaliá-las, buscar possíveis alternativas de solução e, em seguida, fazer a escolha entre alternativas (GOMES, GOMES, ALMEIDA, 2006).

Em relação ao transporte, a decisão multicritério tem sido muito utilizada. Alguns exemplos são apresentados: agrupamento e otimização de rotas de pedestres para o planejamento de rotas multimodais (HOCHMAIR, 2008); busca multicêntrica para transporte de pessoas com deficiência (LEHUÉDÉ et al., 2014). Medda e Nijamp (2003) propuseram uma metodologia de avaliação combinatória para análise complexa de política de transporte usando técnicas de tomada de decisão multicriterios. Bristow e Nellthorp (2000) avaliaram rotas para um Projeto de Transporte na União Européia, assim como De Brucker, Macharis e Verbeke (2011) em seu estudo de multianálise de critérios na avaliação de projetos de transporte. Stott et al. (2011) estudaram um layout automático do mapa do metrô usando otimização multicritério. Seunglim e Seongkwan (2006) utilizaram um processo de tomada de decisão baseado na AHP para a construção de modelo de transporte público em estudo de caso de Jeju, Korea. Uma análise de decisão de múltiplos critérios foi realizada no planejamento integrado para o desenvolvimento do transporte público e uso da terra em Klang Valley, Malásia (SHARIFI et al., 2006). Já Bielli, Carotenuto e Gastaldi (1996) desenvolveram Modelo de Avaliação de Criptografias de Redes de Transporte Público, enquanto Ondrus e Pigneur (2006) fizeram um quadro de avaliação multi-stakeholder de critérios múltiplos de pagamentos móveis, ilustrando a indústria suíça de transportes públicos. Para decisões de infraestrutura, cita-se o trabalho de Gercek, Karpak e Kilncaslan (2004) sobre avaliação de redes de transporte ferroviário alteradas, considerando diferentes modos de transporte.

A problemática utilizada nesta pesquisa caracteriza-se como de ordenação e de escolha. Esta classificação deve-se a que, no estudo, é analisado um conjunto de alternativas, estas alternativas são hierarquizadas segundo uma ordem, para, então, ser escolhida a melhor alternativa.

\subsection{Métodos Multicritério}

Os métodos de Análise de Decisão Multicritério (ADM) têm objetivo de avaliar 
as alternativas para solução do problema, incorporando o julgamento de valor do decisor (BORGES; TEIXEIRA, 2011). Os múltiplos objetivos declarados pelos decisores são, então, representados com uso de múltiplos critérios ou atributos (ALMEIDA, 2011).

Os métodos mais difundidos utilizados no campo de transporte são variantes da teoria multi-atributos (AHP, ANP, MAUT, MAVT, SMART, SMARTER, VISA), métodos (PROMETHEE, ELECTRE) e análise de regime (TURCKSIN, BERNARDINI; MACHARIS, 2011). Segundo Macharis e Bernardini (2015) o método mais usado nos artigos analisados, relacionados aos problemas de transporte de forma geral, é o Analytic Hierarchy Process (AHP), desenvolvido por Saaty (1990) ou uma variante de sua tomada de decisão hierárquica estruturada. Da quantidade total de publicações analisadas por Macharis e Bernardini (2015), 33\% gira em torno da aplicação de AHP ou desenvolve uma variante para essa abordagem aplicável no campo do projeto de transporte.

$\mathrm{Na}$ literatura são encontrados vários métodos que foram desenvolvidos para tratamento de problemas com múltiplos objetivos. Eles são divididos em três grandes grupos (ou famílias de abordagens) - reflexo dos princípios de modelagem de preferência. Pode-se distinguir então, a abordagem do critério único de síntese, a abordagem de julgamento interativo e a abordagem de sobreclassificação (ROY, 1985; VINCKE, 1992).

Dentre os métodos referentes ao primeiro grupo - destaca-se a teoria da utilidade multiatributo, MAUT (Multi-Attribute Utility Theory). O MAUT apresenta uma estrutura axiomática e uma lógica compensatória entre os critérios, de modo a se obter uma função de síntese que agregue todos os critérios em uma única função analítica (KEENEY; RAIFFA, 1976).

Já os métodos interativos envolvem o uso de ferramentas computacionais, onde são desenvolvidas etapas alternadas de diálogo e cálculos. A partir da escolha do decisor às questões apresentadas, o modelo pode efetuar uma redução no espaço de alternativas e seguir para a etapa imediata de nova interação (Almeida, 2011).

Em relação aos métodos de sobreclassificação, merece destaque a família de métodos ELECTRE (Elimination Et Choix Traduisant la Réalité) e PROMETHEE 
(Preference Ranking Organization Method for Enrichment Evaluation). Esses métodos são considerados mais flexíveis, sem compensação entre os critérios e que aceitam incomparabilidade entre as alternativas. São baseados na comparação par a par entre as alternativas, explorando uma relação de sobreclassificação (FIGUEIRA, MOUSSEAU; ROY, 2005; BRANS; MARESCHAL, 2005).

Os métodos ELECTRE são baseados em relações de superação. Pela escolha de um dos métodos desta família para solução do caso em estudo, estes métodos são abordados em mais detalhe.

A família de métodos ELECTRE, de origem francesa, tem como objetivo obter um subconjunto de alternativas, priorizadas frente às demais. É dito que este subconjunto sobreclassificam as que não fazem parte dele. Com isso, o número de alternativas a serem exploradas é reduzido, segundo o conceito de dominância de umas sobre outras. Para isso, são utilizados dois índices: o índice de concordância, que mede a vantagem relativa de cada alternativa sobre as outras, e o índice de discordância, que mede a relativa desvantagem de uma sobre as demais.

Os métodos que constituem a família ELECTRE, com a problemática mais adequada para aplicação, são (ALMEIDA, 2011): ELECTRE I (problemática de escolha, utiliza critério verdadeiro); ELECTRE IS (problemática de escolha, utiliza pseudo-critério); ELECTRE II (problemática de ordenação, utiliza critério verdadeiro); ELECTRE III (problemática de ordenação, utiliza pseudo-critério); ELECTRE IV (problemática de ordenação, utiliza pseudo-critério, sem uso de pesos para os critérios); ELECTRE TRI (problemática de classificação, utiliza pseudo-critério).

Segundo Almeida (2011) os métodos da família ELECTRE são aplicados em duas fases. Inicialmente é construída a relação de sobreclassificação, onde se estabelece uma comparação par a par de alternativas. Em seguida, explora-se a relação de sobreclassificação, aplicando-se um procedimento para resolver o problema em função da problemática específica a ser abordada.

A justificativa do uso do Método ELECTRE I no modelo proposto neste trabalho decorre de alguns fatores.

Em primeiro lugar, Figueira, Mousseau e Roy (2005) destacam que, os métodos da família ELECTRE são indicados para situações onde o decisor deseja avaliar as alternativas em pelo menos três critérios, sendo difícil agregar todos eles 
em uma única escala comum. Este é o caso deste estudo. Além disso, o ELECTRE adere à racionalidade não compensatória. Esta é uma característica do problema em questão, no qual não é desejado que um mau desempenho de uma variável em algum critério seja compensado por um excelente desempenho em outro.

Finalmente, justifica-se a utilização do ELECTRE I pois, no caso estudado, busca-se reduzir o tamanho do conjunto de alternativas, definindo-se um subconjunto menor de alternativas não dominadas (escolha de alternativas). Outro fator a destacar é que o ELECTRE I é um método de simples aplicação e facilidade de leitura de seus resultados. Estes aspectos permitem que a manipulação dos parâmetros do modelo, possa ser mais facilmente feita pelo decisor, para análise de sensibilidade.

\subsection{ELECTRE I}

Belton e Stewart (2002), apresentam três valores de limites definidos no método, para que as relações de comparação não sejam expressas erroneamente devido às diferenças menos importantes:

- $\quad$ preferência (p) e indiferença (q): para estabelecimento das relações de sobreclassificação) e

- $\quad$ rejeição (veto) (v): para cada critério j de maneira que a alternativa a1 não pode sobreclassificar a alternativa a2, se o desempenho da alternativa a2 exceder o desempenho da alternativa $a_{1}$ naquele critério por um valor maior ou igual ao limiar de veto estabelecido.

O método é desenvolvido em 5 etapas, segundo Belton e Stewart (2002):

Etapa 1: Definição de atributos relacionados com o problema a ser resolvido e apresentação das alternativas de acordo com os seus respectivos valores.

Etapa 2: Os pesos dos critérios de avaliação, para a tomada de decisão, podem ser calculados por meio do método Analytic Hierarchy Process (AHP).

Etapa 3: Cálculo das matrizes de concordância e de discordância: 
- Matriz de Concordância: refere-se a força de coalisão do critério a favor da relação de sobreclassificação aSb; refletindo que existe um subconjunto significativo de critérios que apoiam a afirmativa que a alternativa $\alpha$ é (fracamente) preferível à alternativa $b$;

$$
\begin{aligned}
& C\left(a_{1}, a_{2}\right)=\frac{1}{w} \sum_{j=1}^{M} w_{j} c_{j}\left(a_{1}, a_{2}\right), \text { em que } \sum_{j=1}^{M} w_{j} \\
& c_{j}\left(a_{1}, a_{2}\right)=\left\{\begin{array}{lr}
1 & \text { se } f_{j}\left(a_{1}\right)+q_{j} \geq f_{j}\left(a_{2}\right) \\
0 & \text { se } f_{j}\left(a_{1}\right)+p_{j} \leq f_{j}\left(a_{2}\right) \\
\frac{p_{j}+f_{j}\left(a_{1}\right)-f_{j}\left(a_{2}\right)}{p_{j}-q_{j}} & \text { em outros casos }
\end{array}\right\} ; j=1,2, \ldots, M
\end{aligned}
$$

- Matriz de Discordância: inexistem critérios em que a intensidade da preferência de $b$ em relação à alternativa $\alpha$ ultrapasse um limite inaceitável;

$$
\operatorname{dj}\left(a_{1}, a_{2}\right)=\left\{\begin{array}{lr}
0 & \text { se } f_{j}\left(a_{1}\right)+p_{j} \geq f_{j}\left(a_{2}\right) \\
1 & \text { se } f_{j}\left(a_{1}\right)+v_{j} \geq f_{j}\left(a_{2}\right) \\
\frac{f_{j}\left(a_{2}\right)-f_{j}\left(a_{1}\right)-p_{j}}{v_{j}-p_{j}} & \text { em outros casos }
\end{array}\right\} ; j=1,2, \ldots, M
$$

Etapa 4: Cálculo da matriz de credibilidade $s\left(a_{1}, a_{2}\right)$.

- Cálculo das taxas de credibilidade de concordância, credibilidade de discordância e superioridade.

$$
s\left(a_{1}, a_{2}\right)=\left\{\begin{array}{cc}
c_{j}\left(a_{1}, a_{2}\right), \text { se dj }\left(a_{1}, a_{2}\right) \leq c_{j}\left(a_{1}, a_{2}\right), \forall j \text { em que } j \in J\left(a_{1}, a_{2}\right) \text { é o conjunto } \\
\text { de critérios tal que dj }\left(a_{1}, a_{2}\right)>c_{j}\left(a_{1}, a_{2}\right) \\
c_{j}\left(a_{1}, a_{2}\right) * \prod_{j \in J} \frac{1-\operatorname{dj}\left(a_{1}, a_{2}\right)}{1-c_{j}\left(a_{1}, a_{2}\right)} & \text { em outros casos }
\end{array}\right\}
$$

- Credibilidade da concordância: $\emptyset^{+}\left(a_{1}\right)=\sum_{a_{2} E A} s\left(a_{1}, a_{2}\right)$

- Credibilidade de discordância: $\emptyset^{-}\left(a_{1}\right)=\sum_{a_{2} E A} s\left(a_{2}, a_{1}\right)$

- Taxa de superioridade: $\mathrm{R}\left(a_{1}\right)=\frac{\oint^{+}\left(a_{1}\right)}{\emptyset^{-}\left(a_{1}\right)}$

Etapa 5: Seleção da melhor alternativa. 


\section{O ESTUDO DE CASO E DESENVOLVIMENTO DO MODELO ADM}

O modelo proposto neste trabalho é baseado em um método multicritério de apoio a decisão, o ELECTRE I. Ele foi desenvolvido com o intuito de garantir um tratamento mais adequado à etapa de seleção de rotas mais rentáveis para cobrir uma rede de ônibus urbano, usando dados de uma rede de referência apresentada em Mastrapa, Leal e Pessoa (2017). Neste trabalho, os autores consideraram uma rede de Transporte Público simétrica, onde o tempo de viagem e a distância nos arcos e a demanda entre dois nós são os mesmos, independente da direção de viagem. A representação do problema é mostrada a seguir.

A rede é representada por um grafo não-direcionado $G(\mathrm{~N}, \mathrm{~A})$ onde os nós em $\mathrm{N}=\left\{\mathrm{n}_{1}, \ldots, \mathrm{n}_{n}\right\}$ representam interseções na rede viária ou os pontos de demanda das diferentes zonas que compõe a rede em estudo, e as arestas em $A=\left\{a_{1}, \ldots, a_{m}\right\}$ representam as ligações da rede. Uma rota pode, então, ser representada por um caminho na rede de Transporte Público, $\lambda_{l}=\left(n_{1}, n_{2}, \ldots, n_{p}\right)$, onde $n_{i} \in N$. Portanto, a solução para o PPRTPU é especificada por um conjunto de rotas $R=\left\{\lambda_{l}: 1 \leq \mid \leq z\right\}$, onde $z \in \mathbb{Z}^{+}$. Será usado o termo rota para designar uma linha componente da rede de ônibus.

Com o intuito de responder às exigências tanto dos usuários, quanto dos operadores, foi criado o seguinte modelo de PPRTPU, onde as funções objetivo são as seguintes:

Minimizar o tempo total de viagem efetuado por todos os passageiros (TTP) que viajam da sua origem até o respectivo destino.

Minimizar TTP $=\sum d_{i j} * t_{i j} \quad \forall i j \in N^{2}$

Onde $\mathrm{d}_{i j}$ é a demanda de viagens entre o nó $i$ e o nó $j$ e $t_{i j}$ é o menor tempo de viagem entre o nó $i$ e o nó $j$.

Minimizar o número de transbordos (NT) feitos pelos usuários desde o ponto de origem até o destino.

Minimizar NT= $\sum d_{i j} * t r_{i j} \quad \forall i j \in T I$ 
Sendo:

TI: conjunto de trajetos indiretos;

trij $_{\text {: }}$ número de transbordos sobre o trajeto ij.

Ambas as funções objetivas estão sujeitas às seguintes restrições.

VSK $\lambda \geq r \quad \forall x \in F$

Onde:

F: conjunto de rotas candidatas;

r: taxa compatível com a rentabilidade de uma rota;

VSK $\boldsymbol{\lambda}$ : Número de Passageiros por quilometro $=U_{\boldsymbol{\lambda}} / \mathbf{l}_{\boldsymbol{\lambda}}$

Ud: Demanda potencial da rota;

|ג: comprimento da rota;

A rede deste estudo está dividida por zonas. As zonas são áreas básicas, assumidas de tal forma que tenham uma uniformidade com respeito ao uso de solo. O tamanho da zona deve ser considerado de modo que o usuário possa caminhar de qualquer ponto até o nó centroide. Os nós centroides representam cada uma das zonas.

Para o período do estudo - estabelecido de uma hora, a distribuição das viagens entre as zonas é considera conhecida e é representada pela matriz OrigemDestino (O-D). Destaca-se que esta matriz é independente do conjunto de rotas. A demanda com origem ou destino de qualquer parte da zona é assumida como gerada no centroide que representa a zona.

Este modelo teve sua solução por Mastrapa, Leal e Pessoa (2017), baseada na heurística proposta por Aquino (1980). O objetivo era o de achar conjunto mínimo de rotas que atendessem todos os pontos de demanda de uma rede de transporte público urbano.

Assim, tanto Aquino (1980) quanto Mastrapa, Leal e Pessoa (2017) buscaram definir um grupo de rotas para atender todos os pontos de demanda da rede. Os procedimentos consideraram, para avaliar o desempenho das rotas, os indicadores, apresentados na Tabela 1. 
As rotas geradas pelos procedimentos são apresentadas na Tabela 2. $O$ procedimento de Mastrapa, Leal e Pessoa (2017) foi mais eficiente (em tempo de processamento) e eficaz (pelo menor número de rotas) que o de Aquino (1980).

Tabela 1 - Indicadores de desempenho das rotas

\begin{tabular}{ll}
\hline $\mathbf{l}_{\boldsymbol{\lambda}}(\mathbf{k m})$ & Comprimento do conjunto de rotas $\lambda$ \\
\hline $\operatorname{TEMP}_{\boldsymbol{\lambda}}(\mathrm{h})$ & Tempo de viagem (ida e volta) no conjunto de rotas $\lambda$ \\
$\mathbf{U}_{\boldsymbol{\lambda}}$ (unid.) & Demanda potencial do conjunto de rotas $\lambda$ \\
Ca.TMaC (unid.) & Capacidade do trecho mais carregado do conjunto de rotas $\lambda$ \\
Ca.TMeC (unid.) & Capacidade do trecho menos carregado do conjunto de rotas $\lambda$ \\
\hline Fonte: Autores &
\end{tabular}

Tabela 2 - Rotas obtidas pelo método original (AQUINO, 1980) e pelo método modificado (MASTRAPA; LEAL; PESSOA, 2017)

\begin{tabular}{|c|c|c|c|c|}
\hline \multicolumn{3}{|c|}{ LEAL; PESSOA, 201/) } & \multicolumn{2}{|c|}{ (contınua) } \\
\hline No. & $\begin{array}{c}\text { Rotas geradas para o conjunto } \boldsymbol{F} \\
\text { originalmente }\end{array}$ & $\begin{array}{l}\text { Núm. } \\
\text { de nós }\end{array}$ & $\begin{array}{c}\text { Rotas geradas para o conjunto } \\
\text { F modificado }\end{array}$ & $\begin{array}{l}\text { Núm. } \\
\text { de nós }\end{array}$ \\
\hline 1 & $1-22-23-26-10-9-7-8$ & 8 & $1-22-4-24-25-7-8-33-11$ & 9 \\
\hline 2 & $1-19-2-29-28-15-32-16-17-33-13$ & 11 & $1-22-23-26-27-30-31-12-17-33-13$ & 11 \\
\hline 3 & $2-29-28-15-32-16-17-33-8-7$ & 10 & $1-22-4-24-25-7-8-33-13$ & 9 \\
\hline 4 & 2-29-28-27-26-10-9-7 & 8 & $1-19-2-29-28-15-32-16$ & 8 \\
\hline 5 & $2-19-1-22-4-24-25-7-8$ & 9 & $1-22-4-24-25-7-8-33-17$ & 9 \\
\hline 6 & $2-19-1-22-23-26-10-9$ & 8 & $2-19-1-22-4-5-36-6-18-11-33-17-12$ & 13 \\
\hline 7 & $2-19-1-22-4-24-25-7-9$ & 9 & $2-19-1-22-4-24-25-7-9-10-30-15$ & 12 \\
\hline 8 & $2-29-28-15-30-31-12-38-10-9$ & 10 & $2-19-1-22-4-24-25-7-8$ & 9 \\
\hline 9 & $2-29-28-15-30-10-9$ & 7 & $2-19-1-22-23-26-10-9-7-8-33-17-12$ & 13 \\
\hline 10 & $2-19-1-22-23-26-10-38-12$ & 9 & $2-29-28-27-30-31-12-17-33-13$ & 10 \\
\hline 11 & $2-29-28-27-26-10-38-12$ & 8 & $2-29-28-15-32-16-17-33-13$ & 9 \\
\hline 12 & $2-29-28-15-32-16-17-12$ & 8 & $2-19-1-3-37-36-6-18-14$ & 9 \\
\hline 13 & $2-29-28-15-30-31-12-17-33-13$ & 10 & $3-20-21-4-5-36-6-18-11-33-17-12$ & 12 \\
\hline 14 & $2-19-1-3-37-36-6-18-14$ & 9 & $3-1-22-4-24-25-7-8$ & 8 \\
\hline 15 & $2-19-1-22-4-5-36-6-18-14$ & 10 & $3-1-22-23-26-10-9-7-8-33-17-12$ & 12 \\
\hline 16 & $2-29-28-15-30-31-12-17-33-11-18-14$ & 12 & $3-1-22-23-26-10-9-7-8-33-13$ & 11 \\
\hline 17 & $3-1-22-4-5$ & 5 & $3-37-36-6-18-11-33-17-12$ & 9 \\
\hline 18 & $3-1-22-4-24-25-7-8$ & 8 & $3-37-36-6-18-11-33-13$ & 8 \\
\hline 19 & $3-1-22-23-26-10-38-12$ & 8 & $3-1-19-2-29-28-15$ & 7 \\
\hline 20 & $3-20-21-4-22-23-26-10-38-12$ & 10 & $3-37-36-6-18-11-33-17-16$ & 9 \\
\hline 21 & $3-20-21-4-24-25-7-9-10-38-12$ & 11 & $4-24-25-7-8-33-17-12$ & 8 \\
\hline 22 & $4-5-36-6-18-11-33-17-16$ & 9 & $4-5-36-6-18-14$ & 6 \\
\hline 23 & $5-4-22-23-26-10-9$ & 7 & $4-24-25-7-8-33-17-16$ & 8 \\
\hline 24 & $5-4-22-23-26-10-38-12$ & 8 & $5-4-24-25-7-8$ & 6 \\
\hline 25 & $5-36-6-18-14-35-34-13$ & 8 & $5-4-24-25-7-9$ & 6 \\
\hline 26 & $5-4-22-1-19-2-29-28-15$ & 9 & $5-36-6-18-11-33-13$ & 7 \\
\hline 27 & $5-4-22-23-26-27-28-15$ & 8 & $5-4-22-1-19-2-29-28-15$ & 9 \\
\hline 28 & $5-4-24-25-7-9-10-30-15$ & 9 & $6-36-5-4-22-23-26-10-9-7-8-33-13$ & 13 \\
\hline 29 & $5-4-24-25-7-8-33-17-16-32-15$ & 11 & $9-7-8-33-17-16$ & 6 \\
\hline 30 & $5-4-22-23-26-10-30-15$ & 8 & $10-9-7-8-33-11-18-14$ & 8 \\
\hline 31 & $6-18-11-33-8-7-9$ & 7 & $12-17-33-11-18-14$ & 6 \\
\hline 32 & $7-8-33-13-34-35-14$ & 7 & $12-17-16-32-15$ & 5 \\
\hline 33 & $9-7-8-33-13$ & 5 & $13-33-11-18-14$ & 5 \\
\hline 34 & $9-7-8-33-11-18-14$ & 7 & $2-29-28-15-32-16-17-33-11-18-6$ & 11 \\
\hline 35 & $9-10-38-12-17-33-13-34-35-14$ & 10 & $2-29-28-15-32-16-17-33-8$ & 9 \\
\hline 36 & $1-22-23-26-10-38-12-17-33-11$ & 10 & $2-29-28-27-30-10-9$ & 7 \\
\hline 37 & $1-22-23-26-10-38-12-17-33-13$ & 10 & $2-29-28-15-32-16-17-33-11-18-14$ & 11 \\
\hline 38 & $1-22-23-26-10-38-12-17-16$ & 9 & $3-20-21-4-24-25-7-8$ & 8 \\
\hline 39 & $2-29-28-15-32-16-17-33-11-18-6$ & 11 & $3-20-21-4-24-25-7-9$ & 8 \\
\hline 40 & $2-29-28-15-32-16-17-33-11-18-14$ & 11 & $4-22-23-26-27-28-15$ & 7 \\
\hline 41 & $3-20-21-4-5$ & 5 & $5-36-6-18-11-33-17-16-32-15$ & 10 \\
\hline
\end{tabular}


Tabela 3 - Rotas obtidas pelo método original (AQUINO, 1980) e pelo método modificado (MASTRAPA; LEAL; PESSOA, 2017)

\begin{tabular}{ccccc} 
No. & $\begin{array}{c}\text { Rotas geradas para o conjunto } \boldsymbol{F} \\
\text { originalmente }\end{array}$ & $\begin{array}{c}\text { Núm. } \\
\text { de nós }\end{array}$ & $\begin{array}{c}\text { Rotas geradas para o conjunto } \\
\boldsymbol{F} \text { modificado }\end{array}$ & $\begin{array}{c}\text { Núm. } \\
\text { de nós }\end{array}$ \\
\hline $\mathbf{4 2}$ & $3-20-21-4-24-25-7-8$ & 8 & $6-18-11-33-8-7-9-10$ & 8 \\
$\mathbf{4 3}$ & $3-37-36-6-18-11-33-17-12$ & 9 & $9-10-30-31-12$ & 5 \\
$\mathbf{4 4}$ & $3-37-36-6-18-11-33-13$ & 8 & $10-30-31-16$ & 5 \\
$\mathbf{4 5}$ & $3-1-19-2-29-28-15$ & 7 & - & 4 \\
$\mathbf{4 6}$ & $3-37-36-6-18-11-33-17-16$ & 9 & - & - \\
$\mathbf{4 7}$ & $4-24-25-7-8-33-11$ & 7 & - & - \\
$\mathbf{4 8}$ & $4-24-25-7-8-33-13$ & 8 & - & - \\
$\mathbf{4 9}$ & $5-36-6-18-11-33-17-12$ & 7 & - & - \\
$\mathbf{5 0}$ & $5-36-6-18-11-33-13$ & 10 & - & - \\
$\mathbf{5 1}$ & $5-36-6-18-11-33-17-16-32-15$ & 9 & - & - \\
$\mathbf{5 2}$ & $6-18-11-33-17-12-38-10-9$ & 4 & - & - \\
$\mathbf{5 3}$ & $8-33-17-12$ & 9 & - & - \\
$\mathbf{5 4}$ & $9-10-38-12-17-33-11-18-14$ & 6 & & - \\
$\mathbf{5 5}$ & $9-10-38-12-17-16$ & & & - \\
\hline
\end{tabular}

Fonte: Mastrapa, Leal e Pessoa (2017)

A partir das rotas geradas em Mastrapa, Leal e Pessoa (2017), foram criadas 4 conjuntos de alternativas de rotas que conectam a rede toda, qual seja, dão cobertura a todas as opções de viagem aos passageiros / usuários. Estas 4 rotas são mostradas na Tabela 3, acrescidos os valores de desempenho, conforme indicadores para análise.

Tabela 4 - Alternativas de rotas e desempenho

\begin{tabular}{|c|c|c|c|c|c|}
\hline No. & I $\lambda(\mathrm{km})$ & TEMP $\lambda(\mathbf{h})$ & U ג (unid.) & Ca.TMaC (unid.) & Ca.TMeC (unid.) \\
\hline 1 & 7,2 & 0,65 & 209 & 179,4 & 14,8 \\
\hline 2 & 6,6 & 0,73 & 136 & 110 & 29,8 \\
\hline 3 & 5,7 & 0,63 & 60 & 63,6 & 16,2 \\
\hline Total & 19,5 & 2,01 & 405 & 353 & 60,8 \\
\hline 4 & 7,9 & 0,75 & 114,2 & 92,6 & 9,4 \\
\hline 11 & 4,9 & 0,68 & 148 & 134 & 3,8 \\
\hline 13 & 4,9 & 0,47 & 20,8 & 147,4 & 4,8 \\
\hline 15 & 7,1 & 0,55 & 8,6 & 79,4 & 2,6 \\
\hline Total & 24,8 & 2,45 & 291,6 & 453,4 & 20,6 \\
\hline 5 & 9 & 0,67 & 87,4 & 65,8 & 12 \\
\hline 6 & 9,3 & 0,62 & 116,8 & 179,4 & 18 \\
\hline 7 & 8,4 & 0,8 & 167,8 & 147,4 & 9 \\
\hline 10 & 4 & 0,72 & 15,8 & 11,2 & 3,8 \\
\hline Total & 30,7 & 2,81 & 387,8 & 403,8 & 42,8 \\
\hline 8 & 8,2 & 0,68 & 70 & 48 & 6 \\
\hline 12 & 5,7 & 0,57 & 34,6 & 134 & 9,6 \\
\hline 14 & 3,9 & 0,44 & 118 & 113,2 & 5,8 \\
\hline 17 & 4,8 & 0,56 & 83,6 & 65,8 & 10,8 \\
\hline 18 & 3,5 & 0,62 & 56 & 67,8 & 29,8 \\
\hline 20 & 4,4 & 0,55 & 41 & 65,8 & 19 \\
\hline Total & 30,5 & 3,42 & 403,2 & 494,6 & 81 \\
\hline
\end{tabular}

Fonte: Autores 


\subsection{Aplicação do ELECTRE I, extensão ao método de Mastrapa, Leal e Pessoa (2017)}

A ADM é aplicada para a escolha da rota mais rentável, considerando o desempenho de cada rota alternativa.

Para aplicação da etapa 1 do ELECTRE I foram definidos como atributos os indicadores que avaliam o desempenho de cada uma das rotas, resumidos na Tabela 4.

\begin{tabular}{llllll}
\multicolumn{6}{l}{ Tabela 5 - Atributos as rotas a avaliar } \\
\hline No. & $\mathbf{I}_{\boldsymbol{\lambda}(\mathbf{k m})}$ & TEMP $_{\boldsymbol{\lambda}(\mathbf{h})}$ & $\mathbf{U}_{\boldsymbol{\lambda} \text { (unid.) }}$ & Ca.TMaC (unid.) & Ca.TMeC (unid.) \\
\hline 1 & 19,5 & 2,01 & 405 & 353 & 60,8 \\
2 & 24,8 & 2,45 & 291,6 & 453,4 & 20,6 \\
3 & 30,7 & 2,81 & 387,8 & 403,8 & 42,8 \\
4 & 30,5 & 3,42 & 403,2 & 494,6 & 81 \\
\hline
\end{tabular}

Fonte: Autores

Lembra-se que as alternativas são os 4 conjuntos de rotas que conseguem atender todos os pontos de demanda da rede urbana estudada.

$\mathrm{Na}$ etapa 2 do ELECTRE I são comparadas as alternativas, com uso do AHP, por uma escala de importância relativa entre duas alternativas. Este modo foi proposto por Saaty (1990), sendo o mais utilizado. Consiste em: atribuir valores entre 1 a 9, para determinar a importância relativa de uma alternativa em relação a outra. Assim, são calculados os pesos dos critérios, obtendo os valores, apresentados na Tabela 5.

\begin{tabular}{ll}
\multicolumn{2}{l}{ Tabela 6 - Pesos dos critérios avaliados. } \\
\hline Peso do Critério \\
\hline $\mathrm{I}_{\lambda}(\mathbf{k m})$ & 0,129883515 \\
$\operatorname{TEMP}_{\lambda}$ (h) & 0,129883515 \\
$\mathbf{U}_{\lambda}$ (unid.) & 0,38185001 \\
Ca.TMaC (unid.) & 0,041836267 \\
Ca.TMeC (unid.) & 0,030832406 \\
\hline
\end{tabular}

Fonte: Autores 
Com intuito de considerar incerteza e imprecisão, usualmente inerentes aos dados produzidos por previsões e estimativas, foram calculados os limites de preferência, indiferença e veto, apresentados na Tabela 6.

\begin{tabular}{llll}
\multicolumn{2}{l}{ Tabela 7 - Limites de preferência, indiferença e veto } \\
\hline Critério & Limite de & Limite de Indiferença & Limite de veto (v) \\
& Preferência (p) & $\mathbf{( q )}$ & \\
\hline I $\boldsymbol{\lambda}(\mathbf{k m})$ & 6,72 & 3,36 & 13,44 \\
TEMP $\boldsymbol{\lambda}$ (h) & 0,846 & 0,423 & 1,692 \\
U $\boldsymbol{\lambda}$ (unid.) & 68,04 & 34,02 & 136,08 \\
Ca.TMaC (unid.) & 84,96 & 42,48 & 169,92 \\
Ca.TMeC (unid.) & 36,24 & 18,12 & 72,48 \\
\hline
\end{tabular}

Fonte: Autores

A aplicação da etapa 3 do ELECTRE I, resultou na obtenção das matrizes de concordância e discordância, apresentadas nas Tabelas 7 e 8, conforme geradas no RStudio.

Tabela 8 - Matriz de concordância entre as alternativas

\begin{tabular}{lllll}
\hline & Alternativa 1 & Alternativa 2 & Alternativa 3 & Alternativa 4 \\
\hline Alternativa 1 & 1.0000 & 0.7488 & 0.7488 & 0.7854 \\
Alternativa 2 & 0.2512 & 1.0000 & 0.2512 & 0.3025 \\
Alternativa 3 & 0.2512 & 0.7488 & 1.0000 & 0.5171 \\
Alternativa 4 & 0.2146 & 0.6975 & 0.4829 & 1.0000 \\
\hline
\end{tabular}

Fonte: Autores

Os valores apresentados na matriz de concordância indicam a relativa dominância da alternativa 1 sobre a alternativa 2, para cada par comparado, baseado na importância dos pesos relativos dos atributos de decisão.

Tabela 9 - Matriz de discordância

\begin{tabular}{lllll}
\hline & Alternativa 1 & Alternativa 2 & Alternativa 3 & Alternativa 4 \\
\hline Alternativa 1 & 0.0000 & 0.2839 & 0.1271 & 0.0777 \\
Alternativa 2 & 0.8008 & 0.0000 & 0.6794 & 0.7881 \\
Alternativa 3 & 0.3588 & 0.1568 & 0.0000 & 0.1088 \\
Alternativa 4 & 1.0000 & 0.4266 & 0.6412 & 0.0000 \\
\hline
\end{tabular}

Fonte: Autores

Observa-se pela Tabela 8 que, a alternativa 1 é pior do que a alternativa 2, dado que a matriz de discordância mede o grau em que uma alternativa tem pior desempenho que outra. A essência do índice de discordância é que qualquer superação da alternativa 2 sobre a alternativa 1 indicada pelo índice de 
concordância, pode ser anulada se houver outro atributo para o qual a alternativa 2 supera a alternativa 1 , por pelo menos o limite de veto.

Com os valores associados às alternativas, obtidos pelas matrizes de concordância e discordância é possível combinar essas duas medidas e produzir uma medida do grau de superação. Qual seja, obter-se um índice de credibilidade que avalie a força da afirmação de que a alternativa 1 é pelo menos tão boa quanto a alternativa 2. A Tabela 9 apresenta a matriz de credibilidade, etapa 4 do método de apoio a tomada de decisão aplicado.

Tabela 10 - Matriz de credibilidade

\begin{tabular}{ccccc}
\hline Alternativas & $\mathbf{1}$ & $\mathbf{2}$ & $\mathbf{3}$ & $\mathbf{4}$ \\
\hline $\mathbf{1}$ & 1 & 0,7488 & 0,7488 & 0,7854 \\
$\mathbf{2}$ & 0,066825641 & 1 & 0,107551709 & 0,091899283 \\
$\mathbf{3}$ & 0,215103419 & 0,7488 & 1 & 0,5171 \\
$\mathbf{4}$ & 0 & 0,6975 & 0,335069658 & 1
\end{tabular}

Fonte: Autores

A matriz de hierarquização apresenta os elementos para definir a hierarquia das soluções alternativas. A determinação desta ordem hierárquica é obtida pela razão de superioridade para cada alternativa, representada pela Taxa de superioridade $\left(\mathrm{R}\left(\mathrm{a}_{1}\right)\right)$, como apresentada na Tabela 10.

Tabela 11 - Hierarquização das alternativas

\begin{tabular}{ccccc}
\hline & $\begin{array}{c}\text { Credibilidade } \\
\text { de } \\
\text { concordância }\end{array}$ & $\begin{array}{c}\text { Credibilidade } \\
\text { de } \\
\text { discordância }\end{array}$ & $\begin{array}{c}\text { Taxa de } \\
\text { superioridade }\end{array}$ & Ranking \\
\hline Alternativa 1 & 3,283 & 1,28192906 & 2,560984147 & 1 \\
Alternativa 2 & 1,266276634 & 3,1951 & 0,39631831 & 4 \\
Alternativa 3 & 2,481003419 & 2,191421367 & 1,132143483 & 2 \\
Alternativa 4 & 2,032569658 & 2,394399283 & 0,848885009 & 3 \\
\hline
\end{tabular}

Fonte: RStudio

\subsection{Análise dos resultados}

Obtém-se, por fim, em ordem prioritária que, a alternativa 1 tem dominância sobre as alternativas 2, 3 e 4, posicionando-se no primeiro lugar na hierarquia de preferência. Seguindo a primeira alternativa (1), em ordem de dominância, seguem as alternativas 3, 4 e 2, como mostrado na Figura 1, resultado do RStudio. A origem das setas representa a dominância da alternativa 1 . 


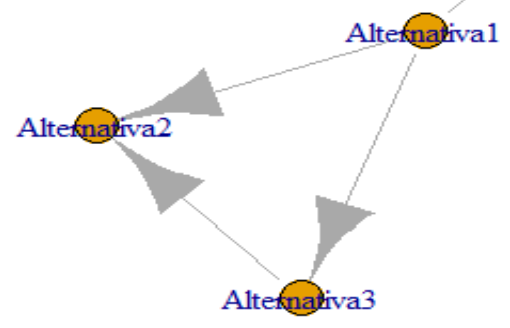

Fonte: RStudio

Resgatando os resultados da tabela 4, destacam-se o desempenho da alternativa 1 que apresenta o menor tempo de comprimento e tempo de viagem, atendendo à maior demanda potencial entre todas as rotas. Em termos de atender ao trecho de maior carga, a alternativa 1 tem menor valor que as demais alternativas e, considerando o trecho de menor carga a alternativa 1 perde apenas para a alternativa 4. Isso pode justificar a segunda posição da alternativa 4.

\section{CONSIDERAÇÕES FINAIS}

O transporte público urbano, para ser efetivo, deveria permitir que os usuários atendessem suas necessidades de mobilidade no tempo desejado, sendo fator de promoção do bem estar social. Para isso o planejamento do transporte público, considerando a definição da capacidade da frota e das rotas para oferta deste serviço, deveria ser de modo integrado ao planejamento do espaço urbano. Porém isso ainda não ocorre nas cidades brasileiras, não apenas devido à complexidade do PPRTPU, como também pelo modo fragmentado como são conduzidas as políticas públicas.

Embora essa questão importante, este trabalho procurou contribuir com o planejamento de transporte urbano. Apresentou-se um modelo para solução de subproblema do PPRTPU para definição de rota que dá cobertura à rede de transporte e que apresenta melhor desempenho em termos de percurso e tempo de 
viagem e atendimento à demanda. Este modelo estendeu um método heurístico, incorporando a ele, a análise multicritério para escolha da rota mais eficiente.

Foi aplicado o método multicritério de apoio a decisão, o ELECTRE I para a seleção e priorização de rotas de ônibus urbanos, considerando critérios de desempenho. A aplicação deste modelo demonstrou ser de fácil utilização e obtenção de resultados.

O método serve de base para a decisão de qual rota priorizar considerando seu desempenho e viabilidade de cobertura de todas as possíveis viagens para mobilidade dos usuários em uma rede de transporte urbana.

A limitação deste trabalho é pelo tamanho da rede de transporte em que o método heurístico foi aplicado. Embora isso, a extensão proposta poderá usar resultados de alternativas de outros problemas para avaliar e escolher a rota mais eficiente, considerando a quilometragem a ser percorrida, o tempo de viagem, a demanda a se atender, considerando a capacidade da frota para atender essa demanda.

\section{REFERÊNCIAS}

ALMEIDA, A.T. O conhecimento e o uso de métodos multicritério de apoio a decisão. 2 edição. Editora Universitária da UFPE, Recife. 2011.

AQUINO, W. J. S. Uma abordagem do problema de definição de rede interurbana de rotas de ônibus. Dissertação (Mestrado em Engenharia de Produção) - Universidade Federal do Rio de Janeiro, Rio de Janeiro, 1980.

ASSOCIAÇÃO NACIONAL DOS TRANSPORTES PÚBLICOS. A integração do transporte público urbano, um procedimento eficiente de organização operacional, está sob suspeita? Revista da ANTP, v. 21, n. 84, p. 82, 1999.

AVELAR, R. B. O impacto do transbordo em sistemas integrados de transporte coletivo por ônibus: Uma análise quantitativa e qualitativa no município de Belo Horizonte. Dissertação (Mestrado em Geotecnia e Transportes) - Universidade Federal de Minas Gerais. Minas Gerais, 2011.

BAAJ, M.; MAHMASSANI, H. An Al-based approach for transit route system. Journal of Advanced Transportation, 25, p.187-209, 1991. https://doi.org/10.1002/atr.5670250205

BELTON, V.; STEWART, T. J. Multiple criteria decision analysis. Kluwer Academic Publishers. London, 2002. https://doi.org/10.1007/978-1-4615-1495-4

BIELLI, M.; CAROTENUTO, P.; GASTALDI, M. Multicriteria evaluation model of public transport networks. In: BIANCO, L., TOTH, P. (Eds.). Advanced methods in transportation analysis. Springer-Verlag, New York, 1996, p.135-156. https://doi.org/10.1007/978-3-642$\underline{85256-57}$ 
BORGES, G.; TEIXEIRA, A. Aplicação do método electre i para seleção de ideias de inovação. In: SIMPÓSIO BRASILEIRO DE PESQUISA OPERACIONAL, 43., 2011. [Anais...]. Ubatuba, 2011.

BRANS, J.P.; MARESCHAL, B. PROMETHEE methods: multiple criteria decision analysis: state of the art surveys. Springer Science + Business Media. New York, p.163-195, 2005. https://doi.org/10.1007/0-387-23081-5 5

BRASIL. Ministério das Cidades. Curso gestão integrada da mobilidade urbana. Módulo II: mobilidade urbana e cidades sustentáveis. Brasília: Semob, nov. 2005, p.26.

BRISTOW, A.L.; NELLTHORP, J. Transport project appraisal in the European Union. Transport Policy, v.7, n.1, p.51-60, 2000. https://doi.org/10.1016/S0967-070X(00)00010-X

CHAKROBORTY, P. Genetic algorithms for optimal urban transit network design. Computer-Aided Civil and Infrastructure Engineering, 18, p. 184-200, 2003. https://doi.org/10.1111/1467-8667.00309

CUNHA, M.J.B. Aplicação da metodologia ELECTRE I de apoio da decisão multicritério na priorização de transporte de mercadoria. In: ENCONTRO NACIONAL DE ENGENHARIA DE PRODUÇÃO, 23., 2003. [Anais...]. Ouro Preto, Minas Gerais, Brasil. Outubro de 2003.

DAGANZO, C. Structure of competitive transit networks. Transportation Research Part B, v. 44, p. 434-446, 2010. https://doi.org/10.1016/j.trb.2009.11.001

DANTZIG, G. B.; HARVEY, R. P.; LANSDOWNE, Z. F.; ROBINSON, D. W.; MAIER, S. F. Formulating and solving the network design problem by decomposition. Transportation Research Part B, v. 13, n. 1, p. 5-17, 1979. https://doi.org/10.1016/0191-2615(79)90003-1

DE BRUCKER, K.; MACHARIS,C.; VERBEKE, A. Multi-criteria analysis and there-solution of sustainable development dilemmas: as take holder management approach. European Journal of Operational Research, v. 224, n.1, p.122-131, 2013.

https://doi.org/10.1016/j.ejor.2012.02.021

FARAHANI, R.Z.; MIANDOABCHI, E. ; SZETO, W.Y.; RASHIDI, H. A review of urban transportation network design problems. European Journal of Operational Research, 229, p. 281-302, 2013. https://doi.org/10.1016/j.ejor.2013.01.001

FIGUEIRA, J.; MOUSSEAU, V.; ROY, B. ELECTRE Methods. Multiple Criteria Decision Analysis: state of the art surveys. Springer Science + Business Media. New York, p.133162, 2005. https://doi.org/10.1007/0-387-23081-5 4

FRIESZ, T.L. Transportation network equilibrium, design and aggregation: key developments and research opportunities. Transportation Research Part A, 19, n. 5-6, p. 413-427, 1985. https://doi.org/10.1016/0191-2607(85)90041-X

GERCEK, H.; KARPAK, B.; KILNCASLAN,T. A multiplecriteria approach for the evaluation of the rail transit networks in Istanbul.Transportation, 31, p.203-228, 2004.

https://doi.org/10.1023/B:PORT.0000016572.41816.d2 
GOMES, L. F. A. M.; GOMES, C. F. S. ; ALMEIDA, A. T. Tomada de decisão gerencial: enfoque multicritério. 2. ed. São Paulo: Atlas, 2006.

GUAN, J.; YANG, H.; WIRASINGHE, S.C. Simultaneous optimization of transit line configuration and passenger line assignment. Transportation Research Part B, v. 40, p. 885-902, 2003. https://doi.org/10.1016/j.trb.2005.12.003

HOCHMAIR, H. H.Grouping of optimized pedestrian routes for multi-modal route planning: a comparison of two cities. Lecture Notes in Geography and Cartography, The European Information Society, p.339-358, 2008. https://doi.org/10.1007/978-3-540-78946818

IBARRA-ROJAS, O.; LÓPEZ-IRARRAGORRI, F.; RIOS-SOLIS, Y. Multiperiod synchronization bus timetabling. Transportation Research Part B, v. 77, p. 38-75, Abril, 2015.

KEPAPTSOGLOU, K.; KARLAFTIS, M. Transit route network design problem: review. Journal of Transportation Engineering, 135, p.491-505, 2009.

https://doi.org/10.1061/(ASCE)0733-947X(2009)135:8(491)

LEHUÉDÉ, F.; MASSON, R.; PARRAGH, S. N.; PÉTON, O.; TRICOIRE, F. A multi-criteria large neighbourhood search for the transportation of disabled people. Journal of the Operational Research Society, v.65, p.983-1000, 2014. https://doi.org/10.1057/jors.2013.17

LOWNES, N.; MACHEMEHL, R. Exact and heuristic methods for public transit circulator design. Transportation Research Part B, v.44, p.309-318, 2010.

https://doi.org/10.1016/j.trb.2009.07.010

MACHARIS, C.; BERNARDINI, A. Reviewing the use of multi-criteria decision analysis for the evaluation of transport projects: time for a multi-actor approach. Transport Policy, v. 37, p.177-186, 2015. https://doi.org/10.1016/j.tranpol.2014.11.002

MAGNANTI, T.L.; WONG, R.T. Network design and transportation planning: models and algorithms. Transportation Science, v.18, n. 1, p. 1-55, 1984.

https://doi.org/10.1287/trsc.18.1.1

MASTRAPA, LORENA HERNÁNDEZ; LEAL, JOSE EUGENIO; PESSOA, LUCIANA. Melhorias em um método heurístico para a solução do problema de desenho de rede de transporte público urbano. Rio de Janeiro, 2017. 102 p. Dissertação (Mestrado) Departamento de Engenharia Industrial, Pontifícia Universidade Católica do Rio de Janeiro. https://doi.org/10.17771/PUCRio.acad.31654

MEDDA, F.; NIJKAMP, P. A combinatorial assessment methodology for complex transport policy analysis. Integrated Assessement, v. 4, n. 3, p. 214-222, 2003.

https://doi.org/10.1076/iaij.4.3.214.23773

ONDRUS, J.; PIGNEUR, Y. A multi-stakeholder multi-criteria assessment framework of mobile payments: an illustration with the Swiss public transportation industry. In: R. SPRAGUE (Ed.). In: HAWAII INTERNATIONAL CONFERENCE ON SYSTEMS SCIENCE, 39., 2006. [Proceedings...]. Kauai, 2006. https://doi.org/10.1109/HICSS.2006.21 
OUYANG, Y.; NOURBAKHSH, S.; CASSIDY, M. Continuum approximation approach to bus network design under spatially heterogeneous demand. Transportation Research Part B, v. 68, 333-344, 2014. https://doi.org/10.1016/j.trb.2014.05.018

RODRIGUES DA SILVA, A, N.; DA SILVA COSTA, M. ;MACÊDO, M. H. Planejamento integrado, organização espacial e mobilidade sustentável no contexto de cidades brasileiras. In: BALBIM, Renato; KRAUSE, Cleandro; LINKE, Clarisse Cunha. Cidade e movimento: mobilidades e interações no desenvolvimento urbano. Brasília: Ipea, 2016. p. 83. Capitulo 4:

ROY, B. Methodologie multicritere d'aide a la decision economica. Paris, 1985.

SAATY, T. L. Multicriteria decision making: the analytic hierarchy process. RWS Publications: Pittsburgh.1990.

SEUNGLIM, K.; SEONGKWAN, M.L. AHP-based decision-making process for construction of public transportation city model: case study of Jeju, Korea. In: JOINT INTERNATIONAL CONFERENCE ON COMPUTING AND DECISION MAKING IN CIVIL AND BUILDING ENGINEERING, 2006. [Proceedings...] Montréal, Canada, 2006.June14-16.

SHARIFI, M.A.; BOERBOOM, L.; SHAMSUDIN, K.B.; VEERAMUTHU, L. Spatial multiple criteria decision analysis in integrated planning for public transport and land use development study in Klang Valley Malaysia. In: ISPRS TECHNICAL COMMISSION SYMPOSIUM, 2., 2006. [Proceedings...]. Vienna, July12-14, 2006, p.85.

STOTT, J.; RODGERS, P.; MARTÍNEZ-OVANDO, J.C.; WALKER, S.G. Automatic metro map layout using multicriteria optimization. IEEETrans.Vis.Comput.Graph, v.17, n.1, p.101114, 2011. https://doi.org/10.1109/TVCG.2010.24

TURCKSIN, L.; BERNARDINI, A.; MACHARIS, C. A combined AHP-PROMETHEE approach for selecting the most appropriate policy scenario to stimulate a clean vehicle fleet. Procedia Social and Behavioral Sciences, 20, 2011, p.945-965.

https://doi.org/10.1016/j.sbspro.2011.08.104

UNITED NATIONS 2014. World urbanization prospects: the 2014 revision population database. United Nations.p.7. Disponível em: http://esa.un.org/unup/. Acesso em: 10 maio 2019.

VINCKE, P. Multicriteria decision-aid. John Wiley \& Sons. Bruxelles, 1992.

WAN, Q.; LO, H. A mixed integer formulation for multiple-route transit network design. Journal of Mathematical Modelling and Algorithms, v. 2, 2003, pp. 299-308. https://doi.org/10.1023/B:JMMA.0000020425.99217.cd

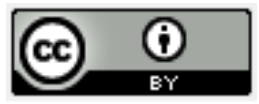

Artigo recebido em: 12/06/2019 e aceito para publicação em: 20/05/2020 DOI: http://dx.doi.org/10.14488/1676-1901.v20i2.3679 